ARTICLE

\title{
IL-33 expression in response to SARS-CoV-2 correlates with seropositivity in COVID-19 convalescent individuals
}

\author{
Michal A. Stanczak (1) 1,9, David E. Sanin (10) 1,9, Petya Apostolova (10 1,9, Gabriele Nerz², Dimitrios Lampaki ${ }^{2}$ \\ Maike Hofmann (10 ${ }^{3}$, Daniel Steinmann ${ }^{4}$, Marvin Krohn-Grimberghe ${ }^{5}$, Robert Thimme ${ }^{3}$, Gerhard Mittler ${ }^{2}$, \\ Cornelius F. Waller (10 6,9凶 , Edward J. Pearce ${ }^{1,7,8,9 凶}$ \& Erika L. Pearce (iD) 1,8,9凶
}

Our understanding of severe acute respiratory syndrome coronavirus 2 (SARS-CoV-2) is still developing. We perform an observational study to investigate seroprevalence and immune responses in subjects professionally exposed to SARS-CoV-2 and their family members (155 individuals; ages 5-79 years). Seropositivity for SARS-CoV-2 Spike glycoprotein aligns with $P C R$ results that confirm the previous infection. Anti-Spike $\operatorname{lgG} / \mathrm{IgM}$ titers remain high 60 days post-infection and do not strongly associate with symptoms, except for fever. We analyze PBMCs from a subset of seropositive and seronegative adults. TLR7 agonistactivation reveals an increased population of IL- $6^{+}$TNF-IL- $1 \beta^{+}$monocytes, while SARS-CoV- 2 peptide stimulation elicits IL-33, IL-6, IFNa2, and IL-23 expression in seropositive individuals. IL-33 correlates with CD4 ${ }^{+} \mathrm{T}$ cell activation in PBMCs from convalescent subjects and is likely due to T cell-mediated effects on IL-33-producing cells. IL-33 is associated with pulmonary infection and chronic diseases like asthma and COPD, but its role in COVID-19 is unknown. Analysis of published scRNAseq data of bronchoalveolar lavage fluid (BALF) from patients with mild to severe COVID-19 reveals a population of IL-33-producing cells that increases with the disease. Together these findings show that IL-33 production is linked to SARS-CoV-2 infection and warrant further investigation of IL-33 in COVID-19 pathogenesis and immunity.

\footnotetext{
${ }^{1}$ Department of Immunometabolism, Max Planck Institute of Immunobiology and Epigenetics, Freiburg, Germany. ${ }^{2}$ Proteomics, Max Planck Institute of Immunobiology and Epigenetics, Freiburg, Germany. ${ }^{3}$ Department of Medicine II, Medical Center-University of Freiburg, Faculty of Medicine, University of Freiburg, Freiburg, Germany. ${ }^{4}$ Occupational Medical Service, Medical Center-University of Freiburg, Faculty of Medicine, University of Freiburg,

Freiburg, Germany. ${ }^{5}$ Department of Cardiology and Angiology I, University Heart Center Freiburg, Freiburg, Germany. ${ }^{6}$ Department of Medicine I, Medical Center-University of Freiburg, Faculty of Medicine, University of Freiburg, Freiburg, Germany. ${ }^{7}$ Faculty of Biology, University of Freiburg, Freiburg, Germany. ${ }^{8}$ Present address: The Bloomberg-Kimmel Institute for Cancer Immunotherapy at Johns Hopkins, Baltimore, MD, USA. ${ }^{9}$ These authors contributed equally: Michal A. Stanczak, David E. Sanin, Petya Apostolova, Cornelius F. Waller, Edward J. Pearce and Erika L. Pearce. ${ }^{凶}$ email: cornelius.waller@uniklinik-freiburg. de; epearce7@jhmi.edu; epearce6@jhmi.edu
} 
S ARS-CoV-2 represents a unique public health challenge in modern times, and as of February 2021 more than $110,000,000$ individuals have been infected worldwide (World Health Organization data). Several studies have shown that SARS-CoV-2 infected individuals seroconvert and develop neutralizing antibodies that are important for protective immunity ${ }^{1-3}$. In addition to the humoral immune response, other work has indicated that both $\mathrm{CD}^{+}$and $\mathrm{CD} 8^{+} \mathrm{T}$ cells with SARS$\mathrm{CoV}-2$ reactivity are present within the blood of convalescent individuals ${ }^{4-6}$.

During acute infection, pro-inflammatory cytokines can contribute to the pathology of SARS-CoV-2-induced acute respiratory disease syndrome, the life-threatening form of this infection. For instance, high serum IL-6, IL-8, TNF, and other cytokines at the time of hospitalization correlated with patient survival ${ }^{7,8}$. Increased cytokine levels were also detected in patients admitted to intensive care units as opposed to less symptomatic individuals ${ }^{9}$. Nevertheless, cytokine levels in SARS-CoV-2 patients appear to be lower than in patients with cytokine release syndrome, sepsis, or influenza ${ }^{10,11}$. Little is known about persisting cytokine signatures in convalescent individuals and how these might relate to humoral and cellular immune responses.

In this study, we set out to investigate seroprevalence and immune responses in subjects professionally exposed to SARSCoV-2 and their family members (155 individuals; ages 5-79 years). We show that after recovery from COVID-19, individuals have persisting, circulating PBMCs that produce IL-33 in response to virus-specific $\mathrm{T}$ cell activation, which correlates with seropositivity. To the best of our knowledge, this is a completely novel finding in the context of COVID-19 and warrants further investigation.

\section{Results and discussion}

We took the opportunity to study a cohort of individuals with a known professional exposure, within a relatively small timeframe ( 2-3 months), as the pandemic was developing. We assessed viral transmission among these individuals and their family members. Specifically, we used an ELISA to measure IgG and IgM titers against the SARS-CoV-2 spike glycoprotein ${ }^{12}$. Our study population included 155 subjects who either tested positive for SARS-CoV-2 via PCR or were in contact with SARS-CoV-2infected individuals. Demographic and disease characteristics of the study population are indicated in Supplementary Table 1. The median age was 36 years and 98 (63.2\%) were females; 99 (64.5\%) had professional exposure to the virus, $44(28.4 \%)$ were exposed within their household and 12 subjects had either multiple or different/unknown exposure sources. Symptoms of upper respiratory tract infection within the last 16 weeks prior to blood collection were reported by 97 (62.6\%) individuals. SARS-CoV-2 PCR testing was performed on 87 individuals, with $47(30.3 \%)$ having a positive PCR result. We evaluated the presence and abundance of antibodies against the spike protein of SARS-CoV-2 in the serum of the study population at a single time point, as well as in 16 historical sera collected prior to the onset of the COVID19 pandemic. We found the highest antibody titers among individuals with a positive SARS-CoV-2 PCR (Fig. 1a), as well as several cases where subjects with a negative PCR result possessed antibodies against the viral protein (9/40). As the date of the PCR and the collection of serum samples were separated by a median time of 49 days, it is possible that these individuals became infected after a PCR was carried out. In addition, we discovered few cases (3/47) where a positive PCR result did not associate with elevated anti-Spike IgG titers.

We used the anti-Spike IgG signal measured in pre-exposure sera to set a threshold to define seropositive cases (Fig. 1a-red dashed line). This resulted in 70 seropositive individuals. We then probed collected sera for IgG and IgM antibodies against the spike protein and its receptor-binding domain (RBD) finding a robust correlation between these measurements (Fig. 1b, c, j, and Supplementary Fig. 1a-e). Seropositive individuals in our cohort were predominantly between $20-60$ years of age (Fig. 1e, f), and were less frequent among 0-20-year-olds (4/16) (Fig. 1e, f), despite documented exposure to infected individuals within their families. We found no association between sex and antibody titers (Fig. 1f, g). Most seropositive subjects reported experiencing symptoms (Fig. 1h). Eight out of 70 subjects who had high antiSpike IgG titers were asymptomatic (Fig. 1h), although anti-RBD IgG titers in some of these individuals fell below the positive threshold (Fig. 1i). To explore possible links between clinical features and antibody titers, we calculated Kendall rank correlation coefficients $(\tau)$ between symptom duration, subject age, and antibody titers in pairs (Fig. 1j). Beyond the strong correlation between IgG and IgM titers for both Spike and RBD, we found modest to no correlation among clinical features. To further explore these associations, we fitted logistic regression models to reported symptoms among seropositive subjects incorporating age, sex, and time between positive PCR and serology as independent variables, setting the symptom report (yes $=1$, no $=0$ ) as the outcome predicted by the antibody titer. Within our cohort, elevated antibody titers against Spike and RBD were reasonable predictors of experiencing fever (Supplementary Fig. $1 \mathrm{~h}-\mathrm{k}$ ), but not for other symptoms. These results might suggest that people with strong immune responses against the virus feel sicker during acute infection, but also have persistent IgM/IgG antibodies as a result of this stronger antiviral response. While previous work $^{1,13}$ has found higher IgM/IgG titers in patients with more severe disease, recent studies highlight the importance of antibody kinetics as a predictor of disease progression ${ }^{14,15}$. It is possible that the comparatively late timepoint of serum sampling, as well as the mild disease severity in our cohort, could explain the discrepancy between those findings and our results. Finally, we observed no appreciable drop in antibody titers in the study population, even 60 days after active infection (Fig. 1k-l and Supplementary Fig. 1f-g).

SARS-CoV-2 represents a unique public health challenge in modern times, with subsequent waves $^{2}$ of infection being an active area of research. While antibodies can neutralize the virus and are therefore likely to be important for protective immunity ${ }^{2}$, it is probable that the pathologic effects of SARS-CoV-2 infection are cell-mediated. The development of IgG antibodies during infection is an indication that a CD4 ${ }^{+} \mathrm{T}$ follicular helper cell response is mounted against this virus ${ }^{16,17}$, and recent work has indicated that both $\mathrm{CD}^{+}$and $\mathrm{CD}^{+}{ }^{+} \mathrm{T}$ cells capable of making IFN- $\gamma$ and TNF are present within the blood of convalescent patients $3,6,17$. We therefore decided to look broadly at cellular immune responses in convalescent patients. We focused on both SARS-CoV-2 Spike protein-specific $\mathrm{T}$ cell responses and innate monocyte responses and measured cytokines known to be produced by both adaptive and innate immune cells. We did this for a subset of 20 convalescent individuals in our cohort, comparing them to 20 seronegative subjects in our study (Supp. Table 2). For all individuals, we assessed a single time point after convalescence. We purified PBMCs from these individuals and examined their composition (Fig. $2 \mathrm{a}-\mathrm{d}$ ) and activation following stimulation (Fig. 2a, e-h), correlating these results to antibody titers (Fig. 2 in bold) using conventional markers (Supplementary Fig. 2) and 2 different stimuli (Fig. 2a). Overall, we did not detect a strong association between the cellular composition, immune cell activation, time from active infection, and antibody titers, (Fig. 2 and Supplementary Fig. 3) with the latter more closely associated with subject age than any other measured parameter 
a

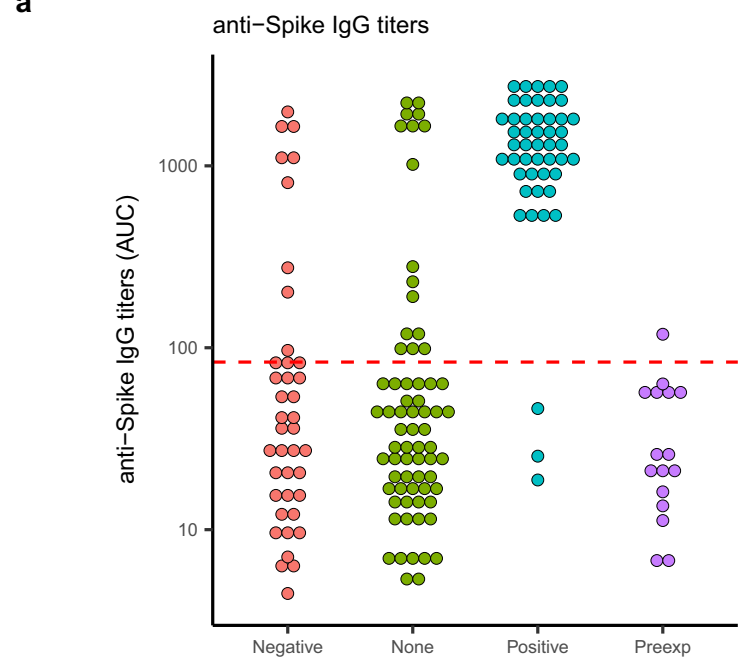

j

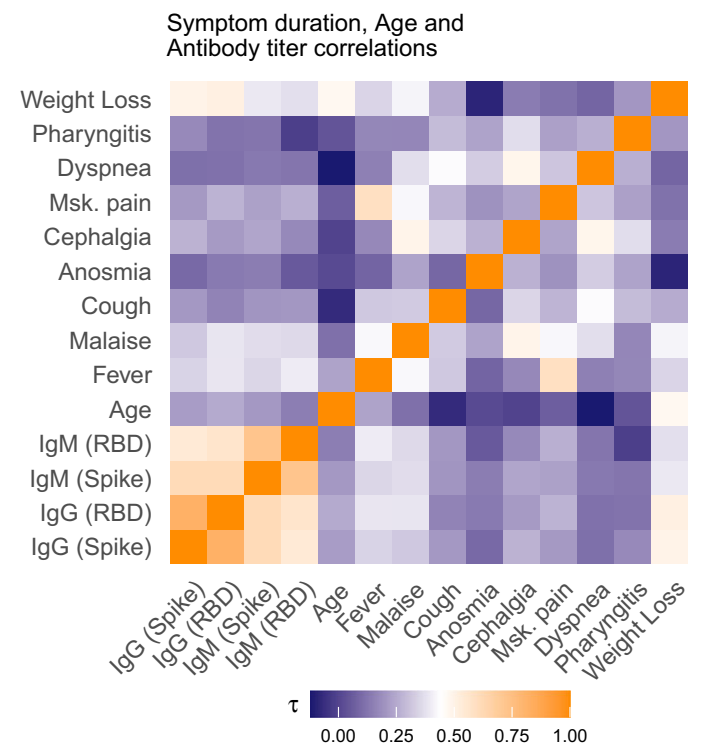

b

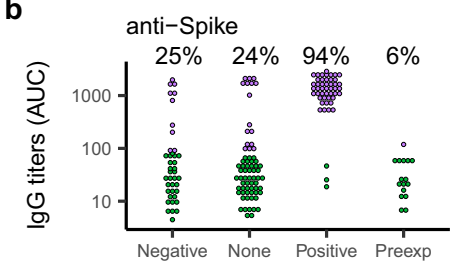

d

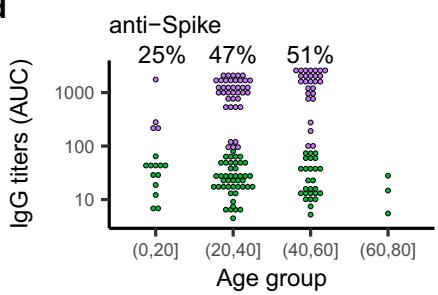

$\mathbf{f}$

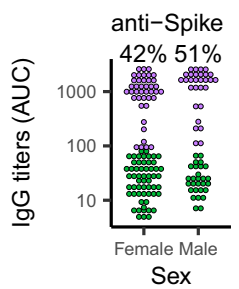

9
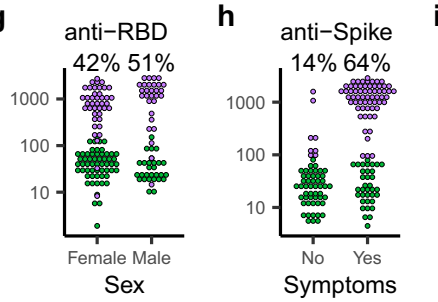

c

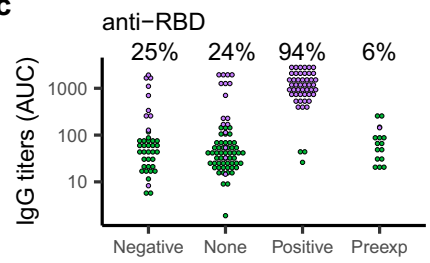

e

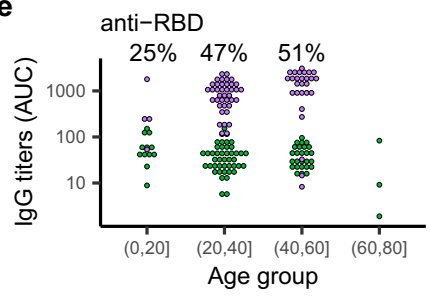

h anti-Spike i anti-RBD

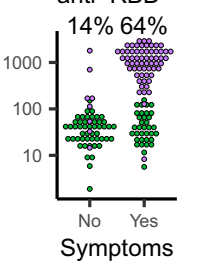

Serology

Seronegative $\bigcirc$ Seropositive

k

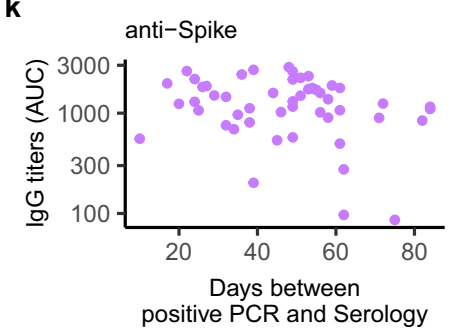

I

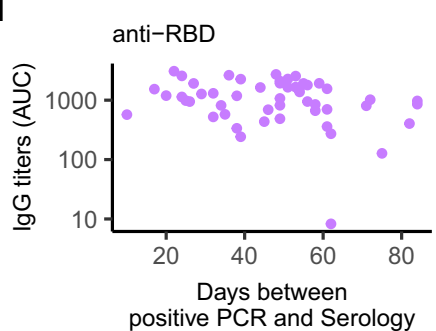

Fig. 1 Serological characterization of the study population. a Anti-Spike IgG titers shown as area under titration curve (AUC) in sera from investigated subjects grouped by SARS-CoV-2 PCR status (Negative-red circles, None-green circles, Positive-blue circles) plus pre-pandemic historical sera (Preexp, magenta circles). The dotted line illustrates the estimated threshold above which subjects were classified as "Seropositive" (mean $+2 x S D-r e d)$. Mean and SD was calculated for all IgG titers below the maximum value measured from Preexp sera $(n=63)$. b-i Anti-Spike $(\mathbf{b}, \mathbf{d}, \mathbf{f}, \mathbf{h})$ and anti-RBD $(\mathbf{c}, \mathbf{e}, \mathbf{g}$, i) IgG titers shown as AUC for investigated subjects grouped by SARS-CoV-2 PCR status (b-c), age group (d-e), sex (f-g) and reported symptoms (h-i). Subjects are colored by anti-Spike lgG serology results (seropositive-purple circles, seronegative-green circles). Percentage seropositive subjects within each category are indicated. $\mathbf{j}$ Detected antibody titers from seropositive subjects were correlated to each other, symptom duration, and subject age using a Kendall rank correlation. A heatmap of the Kendall rank correlation coefficient $(\tau)$ is shown for each pair of variables. $\mathbf{k}-\mathbf{I}$, Anti-Spike (k), and anti-RBD (I) IgG titers shown as a function of time for seropositive subjects. The approximate time of infection was taken as the day of positive SARS-CoV-2 PCR status.

(Fig. 2b-f). However, upon examination of TLR7-driven classical monocyte activation (Fig. $2 \mathrm{~g}$ ), we discovered that the percentage of IL- $6^{+}$TNF-IL- $1 \beta^{+}$monocytes clustered closely with IgG titers. Moreover, SARS-CoV-2 peptide-induced production of IL-33, IL6, IFN-a2, and IL-23 in these cultures (Fig. 2h) also clustered together with IgG titers. Critically, IL-33 production was more closely related to IgG titers than any other measured parameter (Supplementary Fig. 3), including subject age.

While resistance to infection is likely to involve cytokines such as IFN- $\gamma$ produced by T cells, many of the symptoms associated with severe COVID-19 appear to be linked to excessive production of innate cytokines such as IL- 6 and IL- $1 \beta^{18}$. Consequently, we felt that the results highlighting IL-33, IL-6, IFN-a2, IL-23, and IL-1 $\beta$ production (Fig. 2 ) could be important. In line with our clustering analysis, we found that all highlighted features were in fact significantly increased in seropositive subjects (Fig. 3a-e). We reasoned that the observed cytokine production (Fig. 3a-d) should be a result of $\mathrm{T}$ cell activation, as our experimental strategy favors this mechanism. We found that this could be the case for IL-33, which had a strong positive correlation with CD69 expression in $\mathrm{CD}^{+} \mathrm{T}$ cells (Fig. 3f). However, this was not the case for other parameters (Fig. $3 \mathrm{~g}-\mathrm{i}$ ) and not unexpectedly for the percentage of IL- $6^{+} \mathrm{TNF}^{-} \mathrm{IL}-1 \beta^{+}$monocytes (Fig. $3 \mathrm{j}$ ).

To our knowledge, there are no reports associating IL-33 with COVID-19 antibody titers and only one study linking serum IL33 to increased bone marrow precursors in PBMCs of patients infected with SARS-CoV-2 ${ }^{19}$. Given the strong correlation we observed in our data, we decided to explore this result in more 
a

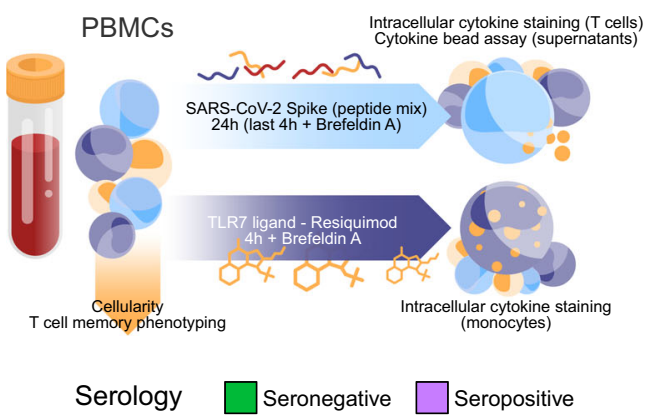

C

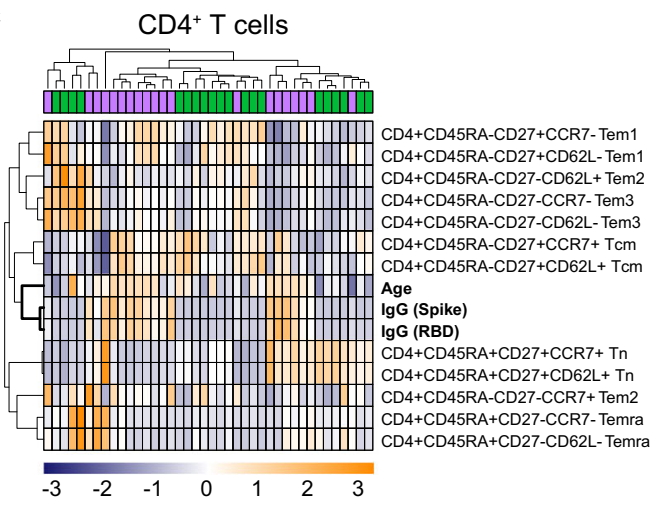

e

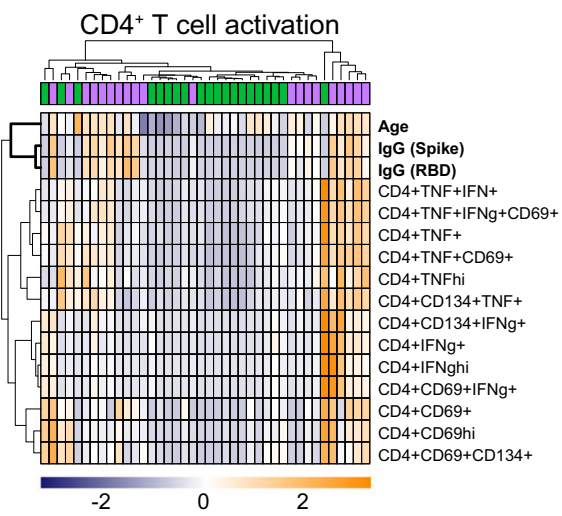

g Classical monocyte activation
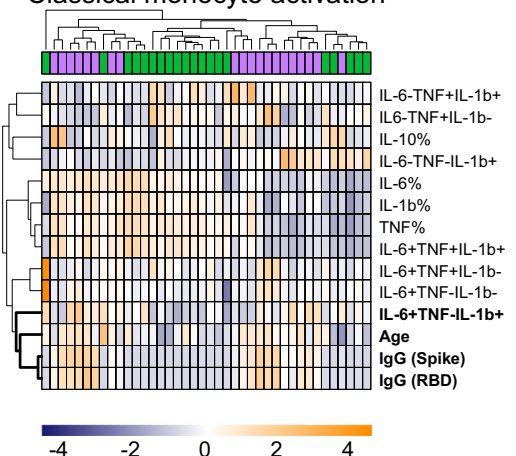

b

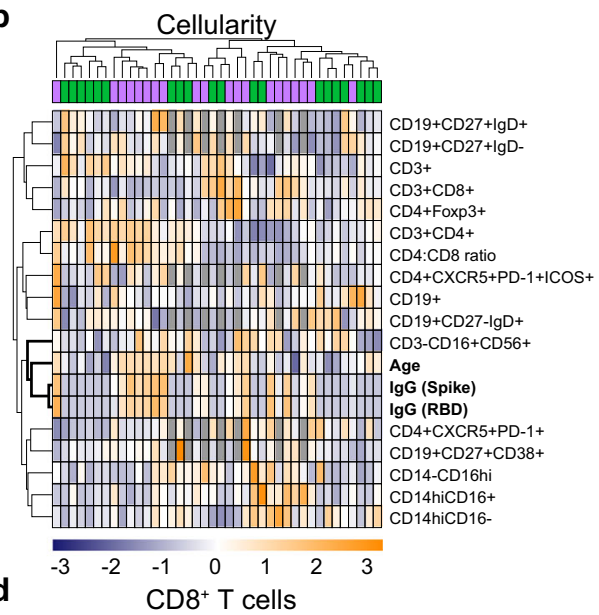

d

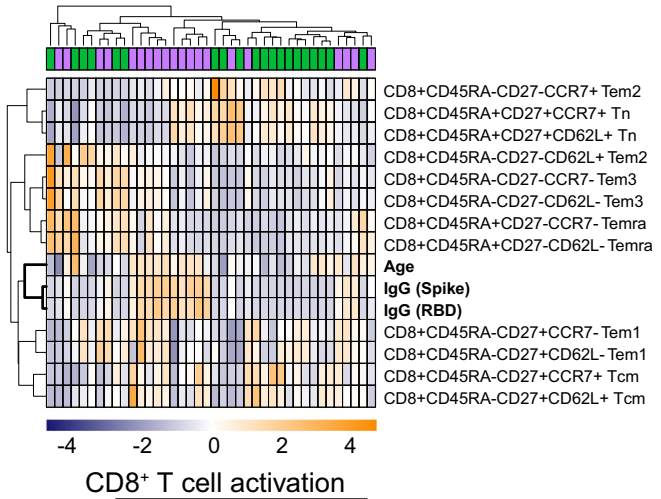

f

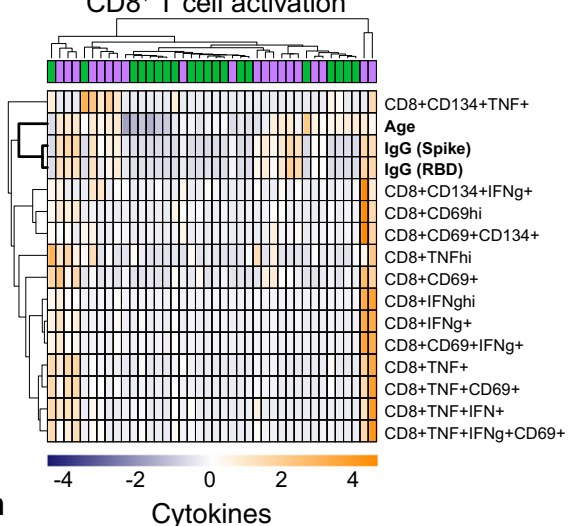

h
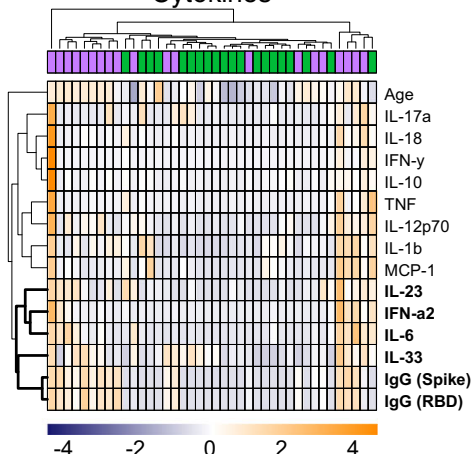

Fig. 2 PBMCs from convalescent and non-infected subjects differ only in innate cytokine production profile. a-h PBMCs in blood collected from a subset of 20 seropositive and 20 seronegative subjects were isolated, analyzed via flow cytometry for cellular composition (b-d), and stimulated with a SARS-CoV-2 spike peptide mix or vehicle control $(\mathbf{e}-\mathbf{f}, \mathbf{h})$, or with TLR7 agonist Resiquimod or vehicle control $(\mathbf{g})$ for indicated times $(\mathbf{a})$. Activation of CD3 ${ }^{+}$CD4 ${ }^{+}(\mathbf{e})$, CD3 ${ }^{+} \mathrm{CD}^{+}+(\mathbf{f})$ and $\mathrm{CD} 14^{h i} \mathrm{CD} 16-$ cells (g) plus cytokine secretion into culture media (h) were measured via flow cytometry. Control corrected (treatment-control) scaled data (where the mean was subtracted from each value then divided by the standard deviation) was then visualized as heatmaps. Scaled anti-Spike and anti-RBD lgG titers plus subject age were included in the results. Data were clustered based on Euclidean distances between features (rows) or subjects (columns), with clusters indicated with dendrograms. Serology results are included for each subject (seropositive-purple; seronegative-green). The closest associations to antibody titers are highlighted in bold letters. Missing values are shown in dark gray. 


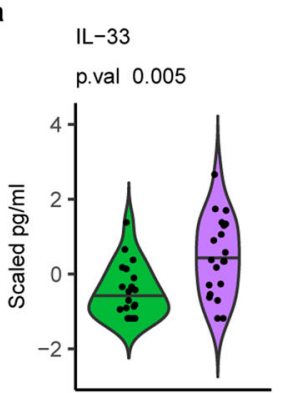

f

$\mathrm{IL}-33$

R.squared 0.6

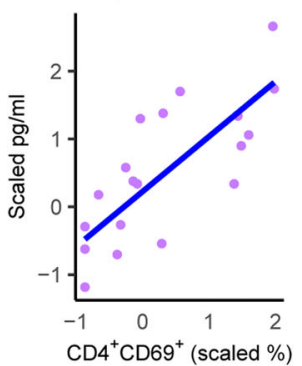

k

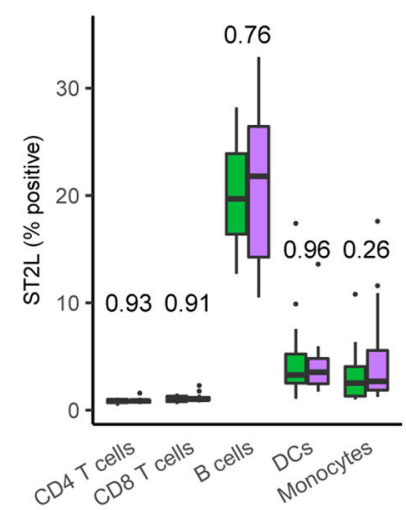

n

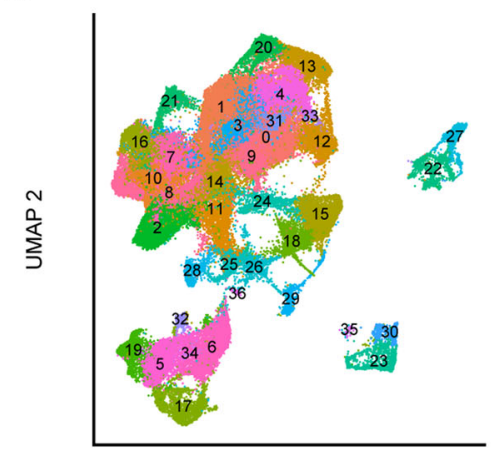

UMAP 1 b IL-6

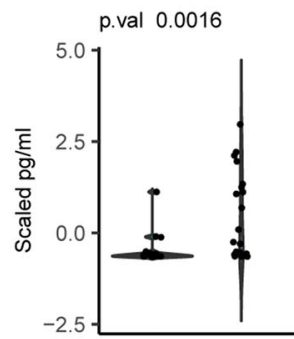

g

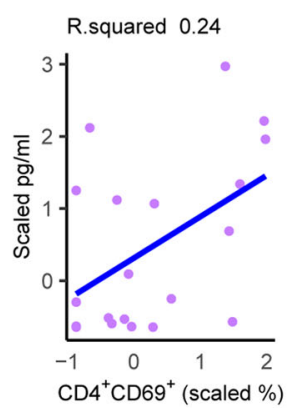

I c

C IFN- $\alpha 2$

p.val 0.0017

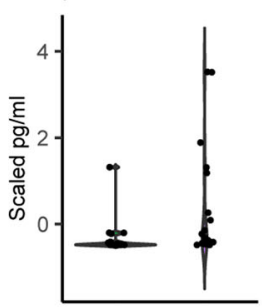

h

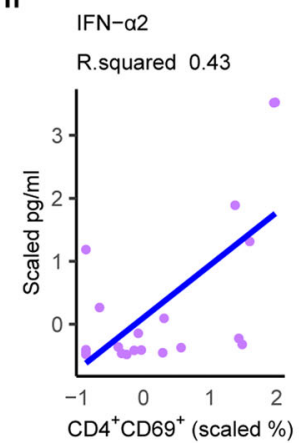

d

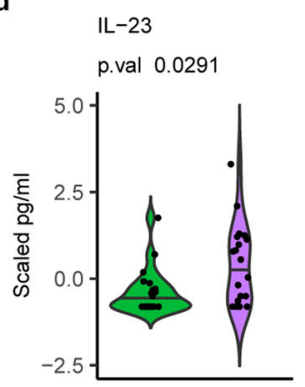

i

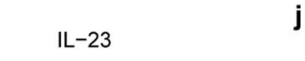

R.squared 0.18

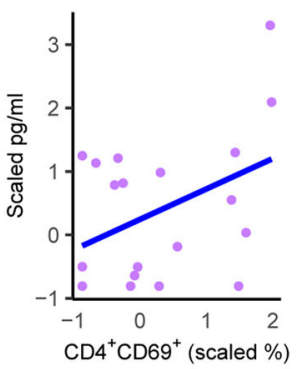

m e

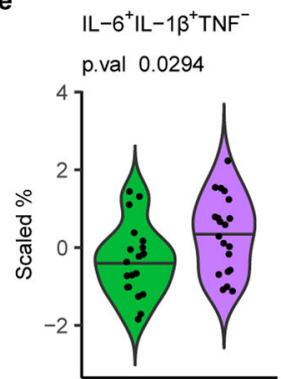

j

$\mathrm{IL}-6^{+} \mathrm{IL}-1 \beta^{+} \mathrm{TNF}^{-}$

R.squared 0.03

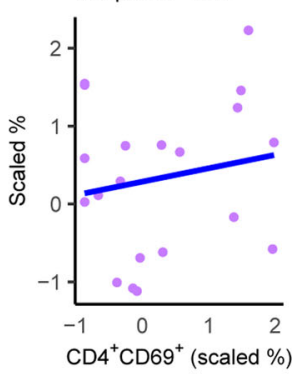

ST2L
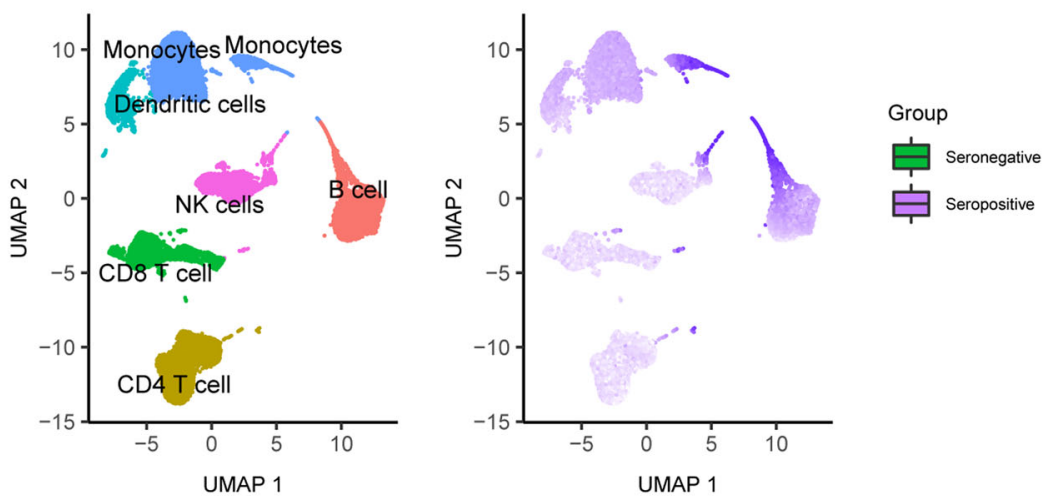

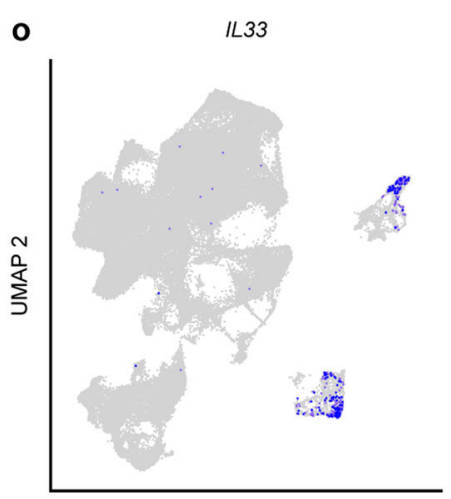

UMAP 1

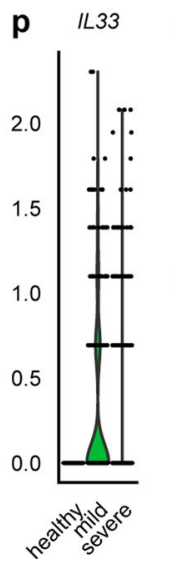

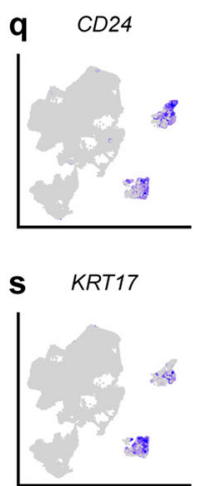

r $\quad C D 59$

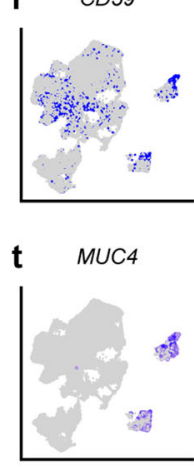

detail. We determined that steady-state serum IL-33 was not altered by previous exposure to SARS-CoV-2 in the individuals of our cohort (Supplementary Fig. 4a), nor was the amount of soluble IL-33 receptor ST2 (sST2) changed in sera from seropositive subjects (Supplementary Fig. 4b). As IL-33 interacts with membrane-bound ST2 (ST2L) on immune cells, we next explored which PBMCs were likely to be influenced by the production of this cytokine. Our results indicate that B cells, and to a lesser extent monocytes and dendritic cells, express the most ST2L, both as a percentage of ST2L $\mathrm{L}^{+}$cells (Fig. 3k) and by MFI (Fig. 3l-m and Supplementary Fig. 4c), making them potential candidates for the regulation exerted by this cytokine. Finally, we retrieved single-cell RNA sequencing data from a recent publication $^{20}$ and processed it to interrogate IL33 expression in 
Fig. 3 IL-33 production correlates with T cell activation and disease severity in SARS-CoV-2 infected subjects. a-e Cytokine production measured in the media of cultured PBMCs (a-d) or via intracellular staining of CD14 ${ }^{\text {hi }} \mathrm{CD} 16^{-}$monocytes from seropositive (purple) and seronegative (green) subjects are shown as scaled values. Two-tailed Mann-Whitney $U$ tests were calculated and the resulting $p$-value is reported. Values for subjects are shown as dots, the sample distribution is presented as a violin plot and the population median is a black line. $\mathbf{f}-\mathbf{j}$ Scaled cytokine production versus corresponding CD4 T cell activation $\left(\mathrm{CD}^{+}{ }^{+} \mathrm{CD} 69^{+}\right)$in PBMC cultures of seropositive individuals are shown. Linear regression curves fitted to these data (blue) alongside $R^{2}$ values are provided. $\mathbf{k}-\mathbf{m}$ ST2L expression as a percentage of positive cells was measured in the indicated cell populations via flow cytometry in PBMCs from seropositive (purple) and seronegative (green) subjects $(n=40)$. Two-tailed Mann-Whitney $U$ tests were calculated and resulting $p$ values are reported. Boxplots indicating the mean (black line), 25th and 75th quantiles (edges), and interquartile ranges (whiskers), as well as outliers (dots), are shown. Flow cytometry staining is presented in a dimensional reduction projection (UMAP), showing immune cell clusters (I) and ST2L median fluorescence intensity $(\mathrm{MFI})(\mathbf{m})$. Scaled MFI is indicated on a gradient from low (gray) to high (purple). $\mathbf{n}$-t Single-cell RNA sequencing data from the bronchoalveolar lavage fluid of 3 healthy individuals compared to 9 SARS-CoV-2 infected subjects with different disease severities ( 3 milds; 6 severe) was retrieved from a public database (GSE145926), grouped into cell clusters (n) and analyzed for the expression of IL-33 (o). Relative expression levels are indicated on a gradient from low (light gray) to high (blue). p IL-33 expression in clusters 23, 27, and 30 presented as a violin plot grouped by disease status. q-t, Key surface (q-r), and lineage-specific (s-t) genes expressed in IL-33 producing cells. Relative expression levels are indicated on a gradient from low (light gray) to high (blue).

the setting of SARS-CoV-2 pulmonary disease (Fig. $3 n-t$ and Supplementary Fig. 4d). These data were generated from cells in BALF of 3 healthy donors and 9 acutely SARS-CoV-2 infected patients, 3 of whom displayed mild symptoms, while the remaining 6 were classed as severely ill (Supplementary Fig. 4d). In these data, we identified IL-33 production by 3 distinct cell clusters (Fig. 3n, o). Strikingly, IL33 expression was confined to SARS-CoV-2 infected patients (Fig. 3 p), concomitant with the increased appearance of the $I L 33^{+}$cell clusters as a result of disease (Supplementary Fig. 4c). IL $33^{+}$cell clusters were most likely stromal cells (Fig. $3 \mathrm{q}-\mathrm{t}$ ) and are unlikely to be responsible for the IL-33 production we observed in PBMC cultures. For this reason, we investigated the source of IL-33 in PBMCs by staining for intracellular IL-33 in conjunction with cell-specific markers, and found that it was most highly expressed in $\mathrm{CD} 14^{+}$monocytes (Supplementary Fig. 5b). However, we observed no differences in the frequency of IL- $33^{+}$cells in PBMC from seropositive vs. seronegative individuals, suggesting that the emergence of COVID-19-specific T cells that are capable of eliciting the release of bioactive IL-33 from other cells that constitutively make this cytokine may be critical. Nevertheless, these clinical data further implicate IL-33 in COVID-19 immunobiology.

Here, we show that after the resolution of infection, an innate immune cytokine signature persists in PBMCs of convalescent subjects, characterized by induction of IL-33 in response to T cell activation with 14-mer peptides, a peptide length suggestive of $\mathrm{CD}^{+} \mathrm{T}$ cell activation ${ }^{21}$. However, the mechanism of activation of the IL-33-producing cells in the PBMCs from convalescent individuals remains unclear at this time. IL-33 was initially described as a cytokine produced as an alarmin by epithelial cells to induce type 2 immune responses ${ }^{22}$, and of interest in this regard, there are reports of eosinophilia, a mark of type 2 immunity, in COVID-19 patients ${ }^{23}$. IL-33 is also linked to lung injury, pulmonary viral infections, and chronic lung diseases ${ }^{24}$. COVID-19 can cause complications such as pneumonia and acute respiratory distress syndrome, as well as invoke lasting damage to the lungs, including lung remodeling and fibrosis, which are consistent with known roles of IL- $33^{25}$. These observations suggest that IL-33 could participate in the pathogenesis of COVID-19, as has recently been discussed in further detail ${ }^{26}$. On the other hand, IL-33 has also been reported to promote antiviral cytotoxic $\mathrm{T}$ cell responses ${ }^{27-29}$ and higher antibody production ${ }^{30,31}$. Thus the presence of a persistent population of cells capable of making IL-33 in response to T cell activation may confer an advantage in the case of secondary exposure. Further work on IL-33 will be needed to elucidate the role of this cytokine in COVID-19.

\section{Methods}

Human subjects. All procedures involving human subjects were approved by the Ethics Committee of the Medical Center-University of Freiburg (305/20). The study was registered at the German Clinical Trial Register (DRKS00022292). Preexposure donor serum samples $(n=16)$ had been collected, aliquoted, and stored at the Medical Center-University of Freiburg prior to June 1st, 2019, with written informed consent for biobank storage obtained from each individual. These individuals were considered to be pre-exposure controls since their biological material had been stored prior to the emergence of SARS-CoV-2. This cohort included $50 \%$ males $(n=8)$ and $50 \%$ females. The median age was 51 years with a range of 39-77 years.

SARS-CoV-2-exposed subjects $(n=155)$ were recruited with a standardized procedure. Individuals who had had a PCR-diagnosed SARS-CoV-2 infection were invited to a COVID-19 aftercare examination by the Occupational Medical Service. Within this examination, individuals were informed about the possibility to participate in the current trial and to also enroll their household members and close contacts who had not been tested for SARS-CoV-2 by PCR or had had a negative PCR result. Inclusion criteria were: contact to SARS-CoV-2-infected individuals, age of at least 5 years, and the ability to provide written informed consent. The exclusion criterium was the presence of symptoms of an acute SARS CoV-2 infection (fever, cough, malaise) within the last 14 days prior to blood collection. Study participants gave their written informed consent prior to study enrollment. For subjects $<18$ years of age, written informed consent was provided by one parent. Collection of biological material was performed at the Medical Center-University of Freiburg. Serum and peripheral blood mononuclear cells (PBMCs) were collected at one or two time points. All samples were de-identified and analyzed in a pseudonymized manner. Information on demographic characteristics and disease course were provided by the subjects using a standardized questionnaire filled at the time point of enrollment. Information on age, sex, PCR testing for SARS-CoV-2, symptoms, symptom duration, treatment, and exposure were analyzed. All individuals $(n=155)$ were included in the 'IgG serology cohort' (Supplementary Table 1), and for these subjects, anti-SARS-CoV2 -Spike and RBD IgG antibody measurements were performed. From this cohort, $n=122$ individuals were included in the 'IgM serology cohort' (Supplementary Table 1), for which anti-SARS-CoV-2-Spike and RBD IgM antibody measurements were performed. According to the results of the antibody measurement, we selected $n=20$ individuals who were seronegative (seronegative cohort) and $n=20$ individuals who were seropositive ('seropositive cohort) for further analysis of PBMCs (Supplementary Table 2). The demographic and clinical characteristics of all cohorts are provided in Supplementary Tables 1 and 2.

Serum asservation and ELISA. Serum was collected in tubes containing clot activators $(7.5 \mathrm{ml})$ and stored at room temperature prior to processing. Tubes were centrifuged at $2500 \times g$ for $10 \mathrm{~min}$ and serum was aliquoted and stored at $-20^{\circ} \mathrm{C}$.

Serology. Collected serum was analyzed for the presence of antibodies against SARS-CoV-2 proteins by adapting a serology assay developed in the laboratory of Dr. Florian Krammer ${ }^{12}$, with some alterations to the protocol. Recombinant spike protein and its receptor-binding domain (RBD) were expressed from mammalian expression plasmids kindly donated by Dr. Krammer ${ }^{12}$. Recombinant proteins were produced employing the Expi293 Expression System (Thermo) following the manufacturer's recommendations. Briefly, cells were grown to exponential phase in serum-free optimized media, then transfected with plasmid-lipid complexes prepared with Expifectamine. Twenty hours later media were supplemented with expression enhancers and finally, media containing recombinant proteins were harvested $72 \mathrm{~h}$ later. Proteins were purified by passing media through Ni-NTA 
Sepharose columns. The purity and identity of recombinant proteins were established by polyacrylamide gel electrophoresis and mass spectrometry.

96-well flat-bottom plates (Corning) were coated with $2 \mu \mathrm{g} / \mathrm{ml}$ of recombinant proteins overnight at $4{ }^{\circ} \mathrm{C}$. Blocking was performed with $200 \mu \mathrm{l} /$ well PBS containing $0.05 \%$ Tween-20 (PBS/T) and 3\% milk for $4 \mathrm{~h}$. Serum samples were diluted 1:50, 1:200,1:800, and 1:3200 performing serial dilutions in PBS/T containing $1 \%$ milk. Serum samples were incubated for $2 \mathrm{~h}$ at room temperature. Plates were washed four times with PBS/T and incubated with an anti-human IgG or IgM secondary antibody (dilution 1:3000, Sigma or Thermo Fisher Scientific) for $1 \mathrm{~h}$ at room temperature. Plates were washed four times with PBS/T and developed using a TMB substrate kit (Biolegend) for $5 \mathrm{~min}$ at room temperature. The colorimetric reaction was stopped by the addition of $2 \mathrm{~N} \mathrm{H}_{2} \mathrm{SO}_{4}$. Internal positive and negative standards were included on each plate to allow for data normalization between different plates. Absorbance at $450 \mathrm{~nm}$ was measured using a TriStar microplate reader (Berthold Technologies).

PBMC collection. Peripheral blood from 20 seronegative and 20 seropositive subjects (based on anti-Spike IgG ELISA) was collected in EDTA-coated tubes and stored at $4{ }^{\circ} \mathrm{C}$ prior to processing. Blood was diluted 1:1 with PBS containing $2 \%$ FCS and $1 \mathrm{mM}$ EDTA. Peripheral blood mononuclear cells (PBMCs) were isolated using a density gradient method. Lymphoprep solution (Stem Cell) was pipetted to the bottom of SepMate-50 tubes (Stem Cell), and diluted blood carefully layered on top. After 10 min centrifugation at $1200 \times g$, the upper phase containing mononuclear cells was transferred to a new tube. PBMCs were washed twice with PBS with $2 \%$ FCS and $1 \mathrm{mM}$ EDTA. After counting, cells were aliquoted, frozen in FCS containing $10 \% \mathrm{DMSO}$, and stored at $-80^{\circ} \mathrm{C}$.

SARS-CoV-2 peptide stimulation. PBMCs were thawed and rested in RPMI containing $10 \%$ FCS, $100 \mathrm{U} / \mathrm{ml}$ penicillin/streptomycin, $4 \mathrm{mM}$ glutamine, and 55 $\mu \mathrm{M} 2$-mercaptoethanol for $2 \mathrm{~h} .4 \times 10^{5} \mathrm{PBMCs}$ were incubated with $2 \mu \mathrm{g} / \mathrm{ml}$ SARSCoV-2 spike PepMix (JPT) or DMSO control in the presence of $2 \mu \mathrm{g} / \mathrm{ml}$ anti-CD28 and $50 \mathrm{U} / \mathrm{ml}$ hIL-2. The PepMix includes a total of 315 14-mer peptides covering the whole spike protein. Incubation was performed for $24 \mathrm{~h}$ with the addition of 5 $\mu \mathrm{g} / \mathrm{ml}$ Brefeldin A (Biolegend) for the last $4 \mathrm{~h}$ to block cytokine secretion. DMSO controls served as negative controls.

TLR7 agonist stimulation. $2 \times 10^{5}$ PBMCs were cultured in RPMI containing $10 \%$ FCS, $100 \mathrm{U} / \mathrm{ml}$ penicillin/streptomycin, and $4 \mathrm{mM}$ glutamine supplemented with 5 $\mu \mathrm{g} / \mathrm{ml}$ Brefeldin A and $5 \mu \mathrm{g} / \mathrm{ml}$ Resiquimod (Invivogen) or water control for $4 \mathrm{~h}$.

Flow cytometry. Prior to cell surface staining, PBMCs were incubated with purified NA/LE Human BD Fc Block (BD, dilution 1:300) and Live/Dead Fixable Aqua or Live/Dead Fixable Blue (both Thermo Fisher, dilution 1:1000) in FACS buffer (2\% FCS and $1 \mathrm{mM} \mathrm{EDTA}$ in PBS) for $20 \mathrm{~min}$ at $4{ }^{\circ} \mathrm{C}$. Cells were then washed and incubated with cell surface antibody cocktails (dilution $1: 200$ ) for $30 \mathrm{~min}$ at $4^{\circ} \mathrm{C}$. Cells were washed with FACS buffer and fixed using BD Cytofix Fixation Buffer (for intracellular cytokine staining) or eBioscience Fixation/Permeabilization kit (for Foxp3 staining) for $20 \mathrm{~min}$ at room temperature. For intracellular staining assays, cells were washed twice with $1 \times \mathrm{BD}$ Perm/Wash buffer or $1 \times$ eBioscience Perm/Wash buffer prior to incubation with intracellular antibody cocktails (dilution 1:200, except for IL-33 antibodies-1:500) for $45 \mathrm{~min}$ at room temperature. Cells were washed twice with $1 \times$ BD Perm/Wash buffer or $1 \times$ eBioscience Perm/ Wash buffer and resuspended in FACS buffer. Flow cytometry data were acquired on a BD LSRFortessa or BD FACSymphony flow cytometer.

IL-33 intracellular flow cytometry staining. Intracellular IL-33 staining was performed on $250000 \mathrm{PBMC} /$ well from two seropositive and two seronegative individuals from the cohort. Cells were stained with cell-surface antibodies and fixed/permeabilized using the BD Cytofix/Cytoperm kit as described above. The recombinant anti-IL-33 antibody (clone 002, PE-conjugated, Abcam) was titrated and used at a final concentration of $0.02 \mu \mathrm{g} / \mathrm{well}$ (dilution 1:500). A recombinant Rabbit IgG monoclonal antibody (clone EPR25A, PE-conjugated, Abcam) was used at the same concentration as an isotype control. The staining was performed for $1 \mathrm{~h}$ at room temperature. As a second approach, the IL-33 monoclonal antibody (clone $6 \mathrm{H} 617$, unconjugated, Invitrogen) was titrated and used at a final concentration of $0.2 \mu \mathrm{g} /$ well (dilution 1:500). The staining was performed for $1 \mathrm{~h}$ at room temperature, followed by incubation with a secondary $\mathrm{PE}$-conjugated rat-anti-mouse antibody (clone A85-1, PE-conjugated, BD Pharmingen, $0.04 \mu \mathrm{g} /$ well) for $30 \mathrm{~min}$. Control staining was performed by incubating only with the secondary PEconjugated antibody.

Cytokine measurement. Supernatants from PBMCs cultured with SARS-CoV-2 PepMix or DMSO control as described above were collected and stored at $-20^{\circ} \mathrm{C}$. Cytokines in culture media were detected using the Legendplex ${ }^{\text {ts }}$ Human Inflammation Panel 1 (Biolegend, Cat\# 740809) following the manufacturer's instructions. This kit allows for simultaneous measurement of IL-1 $\beta$, IFNa2, IFN $\gamma$, TNF, MCP-1, IL-6, IL-8, IL-10, IL-12p70, IL-17A, IL-18, IL-23, and IL-33. Briefly, standards and cell culture supernatants were incubated on a 96-well V-bottom plate with capture beads for $2 \mathrm{~h}$. The plate was washed and biotinylated detection antibodies were added to each well for $1 \mathrm{~h}$, followed by Streptavidin-phycoerythrin (PE) addition for $30 \mathrm{~min}$. Samples were washed and resuspended in FACS buffer. All steps were carried out at room temperature. Results were obtained using a BD LSRFortessa and a BD FACSymphony A5 flow cytometers and FACSDiva (v. 9.0). Data analysis was performed in FlowJo (v. 10.7). Cytokines were identified based on the size and internal dye of the beads. Cytokine concentration in samples was derived from the geometric mean fluorescence intensity for PE interpolated from standard curves calculated from 8 standard controls measured in duplicate.

IL-33 and sST2 were measured in the serum of subjects from our cohort with commercial kits following the manufacturer's instructions (Sigma and ThermoFisher, respectively). Briefly, for IL-33 measurement, standards and samples were incubated for $2.5 \mathrm{~h}$ at room temperature in pre-coated plates. Plates were then washed 4 times and biotinylated detection antibodies were added for $1 \mathrm{~h}$ Following incubation, plates were washed and Streptavidin-HRP was added to each well for $45 \mathrm{~min}$. For sST2 measurement, standards and samples were incubated for $2 \mathrm{~h}$ at room temperature in pre-coated plates with direct addition of biotinconjugated detection antibodies. Following incubation, plates were washed 4 times with a provided buffer, and Streptavidin-HRP was added to each well. Plates were then incubated for $1 \mathrm{~h}$ at room temperature and washed again 4 times. Finally, for both IL-33 and sST2, bound protein was revealed by adding TMB Substrate to each well. Reactions were stopped after $20 \mathrm{~min}$ with provided Stop Solution and absorbance read at $450 \mathrm{~nm}$ immediately.

Data analysis and statistics. Clinical data, ELISA readings, flow cytometry results, and single-cell RNA sequencing data were processed and analyzed using R (Lucent Technologies), which was also employed to generate graphs. $\mathrm{R}$ packages used included: patchwork (v.1.1.0), reshape2 (v1.4.4), dplyr (v. 0.8.5), CytoExploreR (1.0.8), pheatmap (1.0.12) and ggplot2 (v. 3.3.2).

Antibody titers. Background measurements were subtracted from absorbance readings, which were then batch corrected based on internal standards included on each plate. Batch and background corrected data were then used to calculate the area under the dilution curve (AUC) for each subject. To calculate a seropositivity threshold, a subset of AUC values was extracted, composed of all pre-pandemic sera, as well as those subjects with an AUC lower than the maximum obtained from pre-exposure sera and with no SARS-CoV-2 PCR or with a negative PCR result. Positive cases were defined as being at least 2 standard deviations higher than the mean AUC from this sample subset. All other values were defined as seronegative.

Symptoms, age, and titer associations. The relationship between antibody titers subject age, and symptom duration in days was correlated with one another using Kendall rank correlation and reporting $\tau$ as the correlation coefficient. Only complete pairs of observations were used in correlations and all data was scaled by subtracting the mean of each feature and dividing by the corresponding standard deviation. Antibody titers (Ab) were also used to fit logistic regression models capable of predicting the presence of reported symptoms (yes $=1$, no $=0)(S$ ) incorporating the age $(A)$ and sex $(\mathrm{Sx})$ of the subjects, as well as the time between positive PCR and Serology $(t)$ :

$$
S \sim \mathrm{Ab}+A+\mathrm{Sx}+t
$$

Models that performed significantly better than the null model were examined for the significant associations between antibody titers and symptoms ( $p$-value of less than 0.05 in both conditions). The regression coefficient, the regression coefficien divided by its standard error ( $\mathrm{z}$ ) and $p$-value for $\mathrm{Ab}$ titer are reported. Only subjects with a positive PCR and serology ( $n=51$ in IgG cohort and $n=42$ in IgM cohort) were included in this analysis.

Immunophenotyping clustering and regression. Flow cytometry data were analyzed using FlowJo version 10.7. Flow cytometry results were collected and stimulation controls were subtracted from values obtained for treatments. A signal intensity threshold of 5000 was used for CD69, TNF, and IFN-g to define populations with high expression. Corrected values were subsequently scaled and clustered based on Euclidean distances to antibody titers and subject age, which were similarly scaled. Heatmaps and clustering were obtained by using pheatmap v.1.0.12. To complement clustering analysis, correlations were explored between antibody titers and flow cytometry results using Kendall rank correlation and reporting $\tau$ as the correlation coefficient. Selected features were correlated with one another using a linear regression model $(y \sim x)$ and reporting $\mathrm{R}^{2}$ as an indication of the quality of the fit.

Flow cytometry analysis of ST2L expression in PBMCs was carried out using CytoExploreR (R package version 1.0.8. https://github.com/DillonHammill/ CytoExploreR) for gating and initial processing, then uwot ( $\mathrm{R}$ package version 0.1 .10 https://cran.r-project.org/web/packages/uwot/index.html) for dimensionality reduction.

Single-cell RNA sequencing analysis. The transcriptional profiles of $\sim 90,000$ single cells in bronchoalveolar lavage fluid from 3 healthy individuals and 9 SARS-CoV-2 
infected patients ( 3 with mild and 6 with severe disease) were retrieved from a publicly available repository (GSE145926). Matrices with raw unfiltered read counts for detected features in barcoded cells were downloaded for each subject and subsequently processed, analyzed, and visualized in R using Seurat v. $3^{32}$, removing cells with a high percentage of mitochondrial RNA over total RNA $(>25 \%)$ and with less than 200 detected features, normalizing gene expression data by applying regularized negative binomial regression ${ }^{33}$ and using a Uniform Manifold Approximation and Projection (UMAP) $)^{34}$ as a dimensionality reduction approach. Differentially expressed genes within each cluster and across conditions were determined with Seurat as those with a greater than 1.2 fold change and an adjusted $p$-value of less than 0.05 .

Reporting summary. Further information on research design is available in the Nature Research Reporting Summary linked to this article.

\section{Data availability}

All data reported in this study has been submitted to the publisher and is available for download. Source data are provided with this paper.

\section{Code availability}

No custom code was used to generate graphs or analyze data.

Received: 13 August 2020; Accepted: 10 March 2021;

Published online: 09 April 2021

\section{References}

1. Long, Q.-X. et al. Antibody responses to SARS-CoV-2 in patients with COVID-19. Nat. Med. 26, 845-848 (2020).

2. Huang, A. T. et al. A systematic review of antibody mediated immunity to coronaviruses: kinetics, correlates of protection, and association with severity. Nat. Commun. 11, 4704 (2020).

3. Sekine, T. et al. Robust $\mathrm{T}$ cell immunity in convalescent individuals with asymptomatic or mild COVID-19. Cell 183, 158-168.e14 (2020).

4. Braun, J. et al. SARS-CoV-2-reactive T cells in healthy donors and patients with COVID-19. Nature 587, 270-274 (2020).

5. Le Bert, N. et al. SARS-CoV-2-specific T cell immunity in cases of COVID-19 and SARS, and uninfected controls. Nature 584, 457-462 (2020).

6. Peng, Y. et al. Broad and strong memory $\mathrm{CD} 4^{+}$and $\mathrm{CD} 8^{+} \mathrm{T}$ cells induced by SARS-CoV-2 in UK convalescent individuals following COVID-19. Nat. Immunol. 21, 1336-1345 (2020).

7. Del Valle, D. M. et al. An inflammatory cytokine signature predicts COVID19 severity and survival. Nat. Med. 26, 1636-1643 (2020).

8. Burke, H. et al. Inflammatory phenotyping predicts clinical outcome in COVID-19. Respir. Res. 21, 245 (2020).

9. Huang, C. et al. Clinical features of patients infected with 2019 novel coronavirus in Wuhan, China. Lancet 395, 497-506 (2020).

10. Leisman, D. E. et al. Cytokine elevation in severe and critical COVID-19: a rapid systematic review, meta-analysis, and comparison with other inflammatory syndromes. Lancet Respir. Med. 8, 1233-1244 (2020).

11. Mudd, P. A. et al. Distinct inflammatory profiles distinguish COVID-19 from influenza with limited contributions from cytokine storm. Sci. Adv. 6, eabe3024 (2020).

12. Amanat, F. et al. A serological assay to detect SARS-CoV-2 seroconversion in humans. Nat. Med. 26, 1033-1036 (2020).

13. Hou, H. et al. Detection of IgM and IgG antibodies in patients with coronavirus disease 2019. Clin. Transl. Immunol. 9, e01136 (2020).

14. Lucas, C. et al. Kinetics of antibody responses dictate COVID-19 outcome. medRxiv 2020.12.18.20248331 (2020)

15. Zohar, T. et al. Compromised Humoral Functional Evolution Tracks with SARS-CoV-2 Mortality. Cell 183, 1508-1519.e12 (2020).

16. Thevarajan, I. et al. Breadth of concomitant immune responses prior to patient recovery: a case report of non-severe COVID-19. Nat. Med. 26, 453-455 (2020).

17. Mathew, D. et al. Deep immune profiling of COVID-19 patients reveals distinct immunotypes with therapeutic implications. Science 369, eabc8511 (2020).

18. Wen, W. et al. Immune cell profiling of COVID-19 patients in the recovery stage by single-cell sequencing. Cell Discov. 6, 31 (2020).

19. Bernardes, J. P. et al. Longitudinal multi-omics analyses identify responses of megakaryocytes, erythroid cells, and plasmablasts as hallmarks of severe COVID-19. Immunity 53, 1296-1314.e9 (2020).
20. Liao, M. et al. Single-cell landscape of bronchoalveolar immune cells in patients with COVID-19. Nat. Med. 26, 842-844 (2020).

21. Chicz, R. M. et al. Predominant naturally processed peptides bound to HLADR1 are derived from MHC-related molecules and are heterogeneous in size. Nature 358, 764-768 (1992).

22. Molofsky, A. B., Savage, A. K. \& Locksley, R. M. Interleukin-33 in tissue homeostasis, injury, and inflammation. Immunity 42, 1005-1019 (2015).

23. Gong, J. et al. Correlation analysis between disease severity and inflammation related parameters in patients with COVID-19: a retrospective study. BMC Infect. Dis. 20, 963 (2020)

24. Werder, R. B. et al. Chronic IL-33 expression predisposes to virus-induced asthma exacerbations by increasing type 2 inflammation and dampening antiviral immunity. J. Allergy Clin. Immunol. 141, 1607-1619.e9 (2018).

25. Guan, W.-J. et al. Clinical characteristics of Coronavirus Disease 2019 in China. N. Engl. J. Med. 382, 1708-1720 (2020).

26. Zizzo, G. \& Cohen, P. L. Imperfect storm: is interleukin-33 the Achilles heel of COVID-19? Lancet Rheumatol. 2, e779-e790 (2020).

27. Villarreal, D. O. et al. Alarmin IL-33 acts as an immunoadjuvant to enhance antigen-specific tumor immunity. Cancer Res. 74, 1789-1800 (2014).

28. Bonilla, W. V. et al. The alarmin interleukin-33 drives protective antiviral CD8 + T cell responses. Science 335, 984-989 (2012).

29. McLaren, J. E. et al. IL-33 augments virus-specific memory $\mathrm{T}$ cell inflation and potentiates the efficacy of an attenuated cytomegalovirus-based vaccine. J. Immunol. 202, 943-955 (2019).

30. Sarkar, S. et al. IL-33 enhances the kinetics and quality of the antibody response to a DNA and protein-based HIV-1 Env vaccine. Vaccine 37, 2322-2330 (2019).

31. Kayamuro, H. et al. Interleukin-1 family cytokines as mucosal vaccine adjuvants for induction of protective immunity against influenza virus. $J$. Virol. 84, 12703-12712 (2010).

32. Stuart, T. et al. Comprehensive integration of single-cell data. Cell 177, 1888-1902.e21 (2019).

33. Hafemeister, C. \& Satija, R. Normalization and variance stabilization of singlecell RNA-seq data using regularized negative binomial regression. Genome Biol. 20, 1-15 (2019).

34. McInnes, L., Healy, J., Saul, N. \& Großberger, L. UMAP: Uniform Manifold Approximation and Projection. J. Open Source Softw. 3, 861 (2018).

\section{Acknowledgements}

The authors would like to thank Yvonne Neumann, Johan Fridén, Florian Krammer, Mauro Corrado, Asifa Akhtar, Bertram Bengsch, Martin Schwemmle, Evelyn Pearce, Constanze Waller, Fabian Hässler, Mehtap Yücel, and Dan Puleston for their contributions to the study. This study was supported by the Max Planck Society, the German Research Foundation (DFG) Leibniz Prize (E.L.P.), and the DFG under Germany's Excellence Strategy (CIBSS EXC-2189 Project ID 390939984). M.A.S. received funding from the Swiss National Science Foundation.

\section{Author contributions}

M.A.S., D.E.S., P.A., C.F.W., E.J.P. and E.L.P designed the research, provided conceptual input, and analyzed the data. M.A.S., D.E.S., P.A., G.N., D.L. and G.M. performed the experiments. P.A., M.K-G., M.H., D.S., R.T. and C.F.W. recruited participants and collected samples plus clinical information. M.A.S., D.E.S., P.A., E.J.P. and E.L.P wrote the manuscript.

\section{Funding}

Open Access funding enabled and organized by Projekt DEAL.

\section{Competing interests}

E.L.P. and E.J.P. are founders of Rheos Medicines. E.L.P. is a SAB member of ImmunoMet Therapeutics. The remaining authors declare no competing interests.

\section{Additional information}

Supplementary information The online version contains supplementary material available at https://doi.org/10.1038/s41467-021-22449-w.

Correspondence and requests for materials should be addressed to C.F.W., E.J.P. or E.L.P.

Peer review information Nature Communications thanks the anonymous reviewers for their contribution to the peer review of this work.

Reprints and permission information is available at http://www.nature.com/reprints

Publisher's note Springer Nature remains neutral with regard to jurisdictional claims in published maps and institutional affiliations. 
(c) (i) Open Access This article is licensed under a Creative Commons Attribution 4.0 International License, which permits use, sharing, adaptation, distribution and reproduction in any medium or format, as long as you give appropriate credit to the original author(s) and the source, provide a link to the Creative Commons license, and indicate if changes were made. The images or other third party material in this article are included in the article's Creative Commons license, unless indicated otherwise in a credit line to the material. If material is not included in the article's Creative Commons license and your intended use is not permitted by statutory regulation or exceeds the permitted use, you will need to obtain permission directly from the copyright holder. To view a copy of this license, visit http://creativecommons.org/ licenses/by/4.0/.

(C) The Author(s) 2021 